\section{Geographic and Racial/Ethnic Variation in Glycemic Control and Treatment in a National Sample of Veterans With Diabetes}

Diabetes Care 2020;43:2460-2468 | https://doi.org/10.2337/dc20-0514

\section{OBJECTIVE}

Geographic and racial/ethnic disparities related to diabetes control and treatment have not previously been examined at the national level.

\section{RESEARCH DESIGN AND METHODS}

A retrospective cohort study was conducted in a national cohort of $1,140,634$ veterans with diabetes, defined as two or more diabetes ICD-9 codes (250.xx) across inpatient and outpatient records. Main exposures of interest included 125 Veterans Administration Medical Center (VAMC) catchment areas as well as racial/ethnic group. The main outcome measure was $\mathrm{HbA}_{1 \mathrm{c}}$ level dichotomized at $\geq 8.0 \%$ ( $\geq 64 \mathrm{mmol} / \mathrm{mol}$ ).

\section{RESULTS}

After adjustment for age, sex, racial/ethnic group, service-connected disability, marital status, and the van Walraven Elixhauser comorbidity score, the prevalence of uncontrolled diabetes varied by VAMC catchment area, with values ranging from $19.1 \%$ to $29.2 \%$. Moreover, these differences largely persisted after further adjusting for medication use and adherence as well as utilization and access metrics. Racial/ethnic differences in diabetes control were also noted. In our final models, compared with non-Hispanic Whites, non-Hispanic Blacks (odds ratio 1.11 [95\% credible interval 1.09-1.14]) and Hispanics (1.36 [1.09-1.14]) had a higher odds of uncontrolled $\mathrm{HBA}_{1 \mathrm{c}}$ level.

\section{CONCLUSIONS}

In a national cohort of veterans with diabetes, we found geographic as well as racial/ ethnic differences in diabetes control rates that were not explained by adjustment for demographics, comorbidity burden, use or type of diabetes medication, health care utilization, access metrics, or medication adherence. Moreover, disparities in suboptimal control appeared consistent across most, but not all, VAMC catchment areas, with non-Hispanic Black and Hispanic veterans having a higher odds of suboptimal diabetes control than non-Hispanic White veterans.

Diabetes affects $>34$ million Americans and is the seventh leading cause of death, and in 2017, diagnosed diabetes had an estimated economic burden of \$327 billion in the U.S. (1,2). However, despite decades of effective treatments for diabetes, control rates for the disease remain below expert-set goals, with non-Hispanic Blacks (NHBs) and Hispanics having suboptimal $\mathrm{HbA}_{1 \mathrm{c}}$ control relative to non-Hispanic
Kelly J. Hunt, ${ }^{1,2}$ Melanie Davis, ${ }^{1}$ John Pearce, ${ }^{2}$ John Bian, ${ }^{1}$ Mark F. Guagliardo, ${ }^{3}$ Ernest Moy, ${ }^{4}$ R. Neal Axon, ${ }^{1}$ and Brian Neelon ${ }^{1,2}$
${ }^{1}$ Charleston Health Equity and Rural Outreach Innovation Center (HEROIC), Ralph H. Johnson VA Medical Center, Charleston, SC

${ }^{2}$ Department of Public Health Sciences, Medical University of South Carolina, Charleston, SC

${ }^{3}$ Data Governance and Analytics, U.S. Department of Veterans Affairs, Washington, DC

${ }^{4}$ Veterans Health Administration Office of Health Equity, Rockville, $M D$

Corresponding author: Kelly J. Hunt, huntke@ musc.edu

Received 13 March 2020 and accepted 9 July 2020

This article contains supplementary material online at https://doi.org/10.2337/figshare.12659453.

The content of this article does not represent the views of the Department of Veterans Affairs or the U.S. government.

(C) 2020 by the American Diabetes Association. Readers may use this article as long as the work is properly cited, the use is educational and not for profit, and the work is not altered. More information is available at https://www.diabetesjournals .org/content/license. 
Whites (NHWs) (3-5). Geography is a wellknown determinant of health, and an improved understanding of the relationships between geographic factors (social and environmental) and diabetes outcomes may lead to targeted interventions $(6,7)$.

Geographic variation in glucose control and diabetes treatment patterns has not previously been examined at the national level (8-11), despite preliminary evidence that geographic factors may affect $\mathrm{HbA}_{1 \mathrm{c}}$ control. A few small studies found that $\mathrm{HbA}_{1 \mathrm{c}}$ testing was lower in rural than in urban areas, especially in the rural South (12-14). In an Oregon study, $\mathrm{HbA}_{1 \mathrm{c}}$ testing rates in areas with Rural Health Clinics were higher than in areas without them and similar to levels in urban areas, suggesting that increased access may improve treatment (14). Moreover, in a previous national study of regional and geographic variations in $\mathrm{HbA}_{1 \mathrm{c}}$ control in veterans with diabetes (5), we found that the odds of poor $\mathrm{HbA}_{1 \mathrm{c}}$ control was higher in rural than in urban veterans. We also found significant broad geographic differences in $\mathrm{HbA}_{1 \mathrm{c}}$ control, with the odds of poor $\mathrm{HbA}_{1 \mathrm{c}}$ control surprisingly lower in the South than in the Northeast (5), despite a higher prevalence of diabetes $(15,16)$. Limitations included the inability to examine detailed geographic patterns at the national level, a focus on only veterans receiving medications for diabetes, and the inability to account for health care access or utilization metrics.

NHBs and Hispanics with diabetes are more likely to have poor glycemic control than NHWs (3-5,17-20). However, few prior studies have examined whether racial/ethnic differences in $\mathrm{HbA}_{1 \mathrm{c}}$ control vary geographically. The determinants of poor glucose control need to be elucidated to more adequately understand why Hispanics and NHBs experience suboptimal diabetes control, even when access to care is not a factor. Racial disparities in access to medical care, use of medical care, and provider behavior are lower in the U.S. Department of Veterans Affairs (VA) than outside the VA (21). Studies have also indicated that quality of care for diabetes is higher inside than outside the VA (22); however, racial/ethnic disparities in diabetes control remain in the VA $(5,11)$.

The overarching objective of this study is to improve understanding of the geographic distribution (i.e., spatial patterning) of diabetes control and treatment among U.S. veterans, which may point the way for improved outcomes and reducing disparities. Here, we sought answers to the following questions: 1) Do rates of metabolic control exhibit geographic patterning or hotspots? 2) Does patterning vary by race/ethnicity? To address these questions, we performed a retrospective analysis of a national cohort of $>1.1$ million veterans who received diabetes care at the VA in 2015. We hypothesized that there would be geographic differences in suboptimal diabetes control and treatment patterns across the nation and that suboptimal control would be more likely in NHBs and Hispanics than in NHWs. We also hypothesized that suboptimal $\mathrm{HbA}_{1 \mathrm{c}}$ control would be related to type and use of diabetes medication as well as to medication adherence.

\section{RESEARCH DESIGN AND METHODS}

\section{Study Population}

A national cohort of 1,140,634 veterans with diabetes was created from patient records within the Veterans Health Administration (VHA) (Supplementary Fig. 1). Patients with two or more ICD-9 codes for diabetes (250.xx) across inpatient and outpatient records were classified as having diabetes. We required patients to have at least one primary care visit and at least one valid $\mathrm{HbA}_{1 \mathrm{c}}$ laboratory result in fiscal year (FY) 2015. $\mathrm{HbA}_{1 \mathrm{c}}$ laboratory values from $3.5 \%$ to $20 \%$ were considered valid, with values outside this range discarded. The cohort was limited to NHW, NHB, and Hispanic patients who are included in the VHA Planning Systems Support Group (PSSG) Geocoded Enrollee Files. The study was approved by our institutional review board and local VA Research and Development committee.

\section{Outcome Measure}

Suboptimal glycemic control defined as $\mathrm{HbA}_{1 \mathrm{c}} \geq 8.0 \%$ ( $\geq 64 \mathrm{mmol} / \mathrm{mol}$ ) was the primary outcome variable $(23,24)$. In secondary analyses, we also defined suboptimal glycemic control using cut points of $\mathrm{HbA}_{1 \mathrm{c}} \geq 7 \%$ ( $\geq 53 \mathrm{mmol} / \mathrm{mol}$ ) and $\mathrm{HbA}_{1 \mathrm{c}} \geq 9 \%$ ( $\geq 75 \mathrm{mmol} / \mathrm{mol}$ ). Glycemic control was assessed as the mean $\mathrm{HbA}_{1 \mathrm{c}}$ value across FY 2015 , with $99 \%$ of patients having $\mathrm{HbA}_{1 \mathrm{c}}$ measured one to five times in the year (median 2).

\section{Primary Covariates}

Covariates included 1) Veterans Administration Medical Center (VAMC) catchment area and 2) race/ethnicity classified as NHW, NHB, and Hispanic. Catchment areas, the geospatial units of analysis, were defined as the outer geographic boundary of counties in the vicinity of each of 125 VAMCs. Every U.S. county was assigned to a catchment area on the basis of which VAMC a plurality of patients from that county routinely received care from FY 2003-2014. In our cohort, $12 \%$ of patients routinely received care outside their assigned catchment area on the basis of county residence. Because there were counties with split utilization, this mismatch was anticipated. Race and ethnicity were obtained from the outpatient and inpatient Medical SAS files and the PatSub race and ethnicity files (25). Race and ethnicity are collected in the VA by patient report. Patients with multiple ( $n=8,737)$, missing $(n=72,264)$, or other race/ethnicity ( $n=34,039)$ were excluded.

\section{Demographics and Comorbidity}

Age, sex, marital status (married or not married), military service-connected disability dichotomized at $50 \%$ (cut point where veterans are exempt from VA copayments), and comorbidity burden were available. The van Walraven algorithm for the weighted sum of Elixhauser comorbidities was applied on the basis of ICD-9 codes $(26,27)$, resulting in a continuous measure of comorbidity burden.

\section{Medication Type and Medication Possession Ratio}

Veterans were classified as taking insulin only, insulin and oral antihyperglycemic agents, or oral antihyperglycemic agents only. Annual medication possession ratio (MPR) was defined as the number of days' supply divided by 365 days (or if deceased, the number of days until death) for each veteran for insulin or oral antihyperglycemic agents (VA classes HS501 or HS502, respectively). MPRs exceeding one were set to one. For patients on multiple oral medications, MPR was calculated by drug class, and the average was taken (28). An MPR of at least $80 \%$ was considered adherent on the basis of studies suggesting that $80 \%$ adherence is needed to attain the full benefits of medication (29). 


\section{Health Care Utilization and Access Metrics}

Outpatient visit frequency, driving distance to nearest VA primary care site, rurality, and primary care site wait time were considered. The number of days a patient had an outpatient encounter was used as an overall metric of health care utilization and access. The variable was categorized to allow nonlinear associations: $1-3$ visits per year, $4-11$ visits per year, $12-23$ visits per year, or $\geq 24$ visits per year. Driving distance to the primary care site and rurality were obtained from the geocoded PSSG files (30). Driving distance was categorized by quartile (Q1 $\leq 6$, Q2 7-12, Q3 13-23, Q4 >23 miles). Rural/urban residence, as defined from the geocoded PSSG files, dichotomized veterans as living in urban or rural and highly rural areas (30). Primary care wait time was assessed for FY 2015 at the VAMC level using publicly available VA data (31).

\section{Statistical Analysis}

We estimated the effect of catchment area and racial/ethnic group on $\mathrm{HbA}_{1 \mathrm{c}}$ control using four logistic regression analyses, with patient-level $\mathrm{HbA}_{1 \mathrm{c}}$ control as the binary outcome. The first three analyses included 1) demographics + comorbidity burden, 2) demographics + comorbidity burden + medication use, and 3) demographics + comorbidity burden + medication use + utilization/access measures. The fourth analysis was performed on a subsample of veterans on insulin, oral medications, or both (71.5\%). This analysis adjusted for model 3 covariates as well as for medication adherence. Each model incorporated a spatial random effect for catchment area to allow the model to capture spatial dependency among outcomes. The spatial random effect was assigned a conditional autoregressive prior in a Bayesian setting to allow for spatial smoothing, borrowing of information across catchments areas, and small area estimation (32). Initially, we considered a spatial random effect for racial/ethnic group to examine whether disparities varied across catchment area (i.e., to examine a "space-by-race" interaction effect). As a sensitivity analysis, we also fit a fixed-effects interaction model for model 3, which included fixed dummy indicators for catchment area as well as race-by-catchment interaction terms. We found no evidence of a space-by-race interaction effect globally $(-2 \log \mathrm{L} P=$ 1.00 for likelihood ratio test comparing models with and without interaction terms), although there were a few pairwise comparisons that reached statistical significance at the $P=0.01$ level for NHBs. Therefore, we excluded interaction terms throughout the analyses.

We performed all analyses in $\mathrm{R}$ software (33) using a Gibbs sampling code we developed specifically to handle large, spatially correlated data (34). For each model, we calculated the posterior probability of suboptimal glycemic control for an "average" patient within each catchment area (i.e., a patient with average covariate values). These probabilities were then mapped to show the geographic distribution of suboptimal glycemic control after controlling for the underlying population distributions as we sought to isolate a spatial effect. We also report posterior odds ratios (ORs) and 95\% Bayesian credible intervals (Cls) for demographic, medication, and access variables. Next, we calculated racial/ethnic group-specific probabilities of suboptimal control for average Hispanic, NHW, and NHB veterans within each VAMC catchment area (Fig. 2B-D). Twelve percent of catchment areas had $<30$ but at least 6 Hispanic patients, and $5 \%$ of catchment areas had $<30$ but at least 10 NHB patients. Low sample sizes did not pose a problem, however, because the smoothing property of the spatial random effect provided robust estimates even for sparse catchment areas. For all analyses, we assumed weakly informative priors for model parameters, which allowed the data to play a dominant role in estimation. For all models, we ran the Gibbs sampler for 5,000 iterations following a burn-in to ensure convergence of the algorithm (34).

\section{RESULTS}

The final cohort consisted of $1,140,634$ veterans with diabetes residing in 125 VAMC catchment areas who received primary care at the VA in FY 2015, of whom 74.8\% were $\mathrm{NHW}, 19.4 \%$ were $\mathrm{NHB}$, and $5.9 \%$ were Hispanic (Table 1). The mean age was 67.7 years, $97.0 \%$ were male, and $28.5 \%$ were not using diabetes medications. Among the 815,255 veterans using diabetes medications, $43.6 \%$ were adherent and $33 \%$ had uncontrolled diabetes $\left(\mathrm{HbA}_{1 \mathrm{c}} \geq 8 \% ; \mathrm{HbA}_{1 \mathrm{c}} \geq 64 \mathrm{mmol} / \mathrm{mol}\right)$, which was higher than the overall population (25.5\%).

\section{Spatial Variation Across Catchment Areas \\ Model 1}

Results from our logistic analyses adjusted for age, sex, race, service-connected disability, marital status, van Walraven comorbidity score, and spatial random effects for catchment areas revealed that the prevalence of uncontrolled diabetes varied by VAMC catchment area, with values ranging from $19.1 \%$ to $29.2 \%$. Figure $1 A$ identifies the geographic distribution of VAMC catchment areas with significantly (diagonal lines) lower and higher odds of uncontrolled diabetes compared with an average catchment area. The map reveals that the prevalence of uncontrolled diabetes varies substantially by catchment. The spatial randomeffect variance was 0.05 (Table 2 ), indicating the presence of unexplained variation. Figure 1 shading reflects adjusted quintile of catchment area odds of uncontrolled diabetes, and the legend reflects the catchment areas with the highest and lowest relative odds of uncontrolled diabetes within each quintile (i.e., relative odds comparing each catchment area with an average catchment area). The ORs for uncontrolled diabetes in catchment areas in the lowest quintile ranged from 0.743 to 0.925 and in the highest quintile, from 1.073 to 1.277 (Table 2, model 1 , and Fig. 1A).

\section{Model 2}

Further adjustment for use and type of diabetes medication (Table 2, model 2, and Fig. $1 B$ ), did not diminish spatial variation in diabetes control (spatial variance 0.05 ). The estimated prevalence of uncontrolled diabetes varied by VAMC catchment area from $15 \%$ to $24 \%$. ORs for uncontrolled diabetes in catchment areas in the lowest quintile ranged from 0.728 to 0.904 and in the highest quintile, from 1.090 to 1.263 .

\section{Model 3}

Additional adjustment for health care utilization and access metrics, including outpatient visit days, driving distance to primary care site, rural residence, and VAMC-level wait time for primary care appointments, only fractionally diminished spatial variation in diabetes control, with catchment-level ORs remaining virtually unchanged (Table 2, model 3, 
Table 1-Cohort characteristics for the overall population, stratified by racial/ethnic group and limited to those using medication to treat their diabetes

\begin{tabular}{|c|c|c|c|c|c|}
\hline & $\begin{array}{c}\text { Overall } \\
(n=1,140,634)\end{array}$ & $\begin{array}{c}\text { NHW } \\
(n=852,704)\end{array}$ & $\begin{array}{c}\text { NHB } \\
(n=220,932)\end{array}$ & $\begin{array}{c}\text { Hispanic } \\
(n=66,998)\end{array}$ & $\begin{array}{c}\text { Medicated* } \\
(n=815,255)\end{array}$ \\
\hline \multicolumn{6}{|l|}{ Demographic } \\
\hline Age (years) & $67.7(10.6)$ & $69.1(10.2)$ & $63.4(10.4)$ & $64.7(11.3)$ & $66.9(10.2)$ \\
\hline Male & 96.96 & 97.56 & 94.56 & 97.26 & 97.07 \\
\hline Service-connected disability $\geq 50 \%$ & 34.48 & 32.33 & 40.54 & 41.97 & 37.16 \\
\hline Married & 60.24 & 63.36 & 48.18 & 60.22 & 58.87 \\
\hline $\begin{array}{l}\text { Comorbidity burden } \\
\text { van Walraven score }\end{array}$ & $1.80(6.35)$ & $1.99(6.23)$ & $1.32(6.79)$ & $0.92(6.08)$ & $1.81(6.52)$ \\
\hline \multicolumn{6}{|l|}{ Medication use and type } \\
\hline No medication & 28.53 & 29.38 & 26.60 & 24.01 & 0 \\
\hline Insulin only & 12.97 & 12.82 & 14.07 & 11.19 & 18.14 \\
\hline Oral medication only & 37.35 & 36.94 & 37.88 & 40.81 & 52.25 \\
\hline Both oral and insulin & 21.16 & 20.86 & 21.45 & 23.98 & 29.61 \\
\hline \multicolumn{6}{|l|}{ Medication adherence } \\
\hline MPR $>80 \% *$ & NA & 46.73 & 34.12 & 36.96 & 43.62 \\
\hline \multicolumn{6}{|l|}{ Utilization and access metrics } \\
\hline $1-3$ & 9.14 & 10.19 & 5.90 & 6.38 & 6.25 \\
\hline $4-11$ & 34.83 & 36.04 & 30.61 & 33.40 & 32.36 \\
\hline $12-23$ & 28.27 & 27.66 & 29.92 & 30.62 & 30.24 \\
\hline$\geq 24$ & 27.76 & 26.12 & 33.56 & 29.60 & 31.15 \\
\hline Distance to primary care (miles) & $16.5(15.5)$ & $18.0(16.1)$ & $11.9(11.5)$ & $13.2(15.4)$ & $16.8(15.7)$ \\
\hline Rural residence & 37.90 & 44.06 & 19.53 & 20.14 & 38.33 \\
\hline Primary care wait time (days) & $4.4(2.4)$ & $4.3(2.3)$ & $5.0(2.8)$ & $4.8(2.2)$ & $4.44(2.5)$ \\
\hline \multicolumn{6}{|l|}{ Primary outcome } \\
\hline Uncontrolled $\mathrm{HbA}_{1 \mathrm{c}} \geq 8 \%(\geq 64 \mathrm{mmol} / \mathrm{mol})$ & 25.48 & 24.04 & $28.9 \% 2$ & 32.51 & 33.00 \\
\hline \multicolumn{6}{|l|}{ Secondary outcomes } \\
\hline Uncontrolled $\mathrm{HbA}_{1 \mathrm{c}} \geq 7 \%(\geq 53 \mathrm{mmol} / \mathrm{mol})$ & 49.78 & 49.36 & 49.67 & 55.36 & 61.50 \\
\hline Uncontrolled $\mathrm{HbA}_{1 \mathrm{c}} \geq 9 \%(\geq 75 \mathrm{mmol} / \mathrm{mol})$ & 12.25 & 10.74 & 16.48 & 17.49 & 16.09 \\
\hline
\end{tabular}

Data are mean (SD) or \%. NA, not applicable. * Limited to those taking medication.

and Fig. 1C). The estimated prevalence of uncontrolled diabetes varied by VAMC catchment area from $16 \%$ to $24 \%$.

\section{Model 4}

Limiting the population to patients using diabetes medication and further adjusting for medication adherence resulted in a similar pattern of uncontrolled diabetes by catchment area (Table 2, model 4, and Fig. 1D).

\section{Secondary Analyses}

Results are presented in Supplementary Table 1 and Supplementary Fig. 2A-D. Analyses were completed using two other commonly accepted cut points for suboptimal diabetes control. An $\mathrm{HbA}_{1 \mathrm{c}}$ cut point of $\geq 7 \%$ (53 $\mathrm{mmol} / \mathrm{mol}$ ) resulted in $49.8 \%$ of veterans with suboptimal diabetes control, while an $\mathrm{HbA}_{1 \mathrm{c}}$ cut point of $\geq 9 \%$ ( $75 \mathrm{mmol} / \mathrm{mol}$ ) resulted in $12.3 \%$ of veterans with suboptimal control. Using either secondary $\mathrm{HbA}_{1 \mathrm{c}}$ cut point to define suboptimal control generally resulted in similar patterns of uncontrolled diabetes by catchment area
(Supplementary Fig. 2A-D), although spatial variance was higher for the $\mathrm{HbA}_{1 \mathrm{c}}$ cut point of $\geq 7 \%$ (53 $\left.\mathrm{mmol} / \mathrm{mol}\right)$ (Supplementary Table 1).

\section{Racial/Ethnic Variations}

Racial/ethnic differences in uncontrolled diabetes were evident in unadjusted analyses, with uncontrolled diabetes being $24.0 \%$ in $\mathrm{NHW}, 28.9 \%$ in $\mathrm{NHB}$, and $32.5 \%$ in Hispanic veterans (Table 1).

\section{Model 1}

After adjusting for demographics, comorbidity burden, and spatial random effects for catchment area, the odds of uncontrolled diabetes were 1.07 (95\% Cl 1.05, 1.09) times higher in NHB and 1.34 (1.31, 1.38) times higher in Hispanic than NHW veterans (Table 2, model 1 ). While both racial/ethnic group and VAMC catchment area were associated with uncontrolled diabetes, we did not find evidence of a space-by-race group interaction. In other words, racial/ethnic disparities in poor glycemic control appeared consistent across most catchment areas on the multiplicative scale (spatial variance of the combined interaction terms was negligible at 0.00005 ).

Figure $2 A-D$ provides probability maps by catchment area for the overall study population and for each racial/ethnic group on the basis of model 1 parameter estimates. To enable comparison across maps, quintile cut points on the basis of the overall population (Fig. $2 A$ ) were applied across Fig. $2 A-D$. In the overall population, the probability of uncontrolled $\mathrm{HbA}_{1 \mathrm{c}}$, adjusted for model 1 covariates, varied by VAMC catchment area (range 19.1-29.2\%). The adjusted probability varied by VAMC catchment area for NHWs (18.7-28.4\%), for NHBs (19.8-29.8\%), and for Hispanics (23.6$34.9 \%)$. The darker shading in the NHB and Hispanic maps relative to the NHW map indicates the increased number of catchment areas having a probability of uncontrolled diabetes of at least $25.7 \%$. Hence, while there was no evidence of a space-by-race group difference on a multiplicative scale, catchment areas 
A

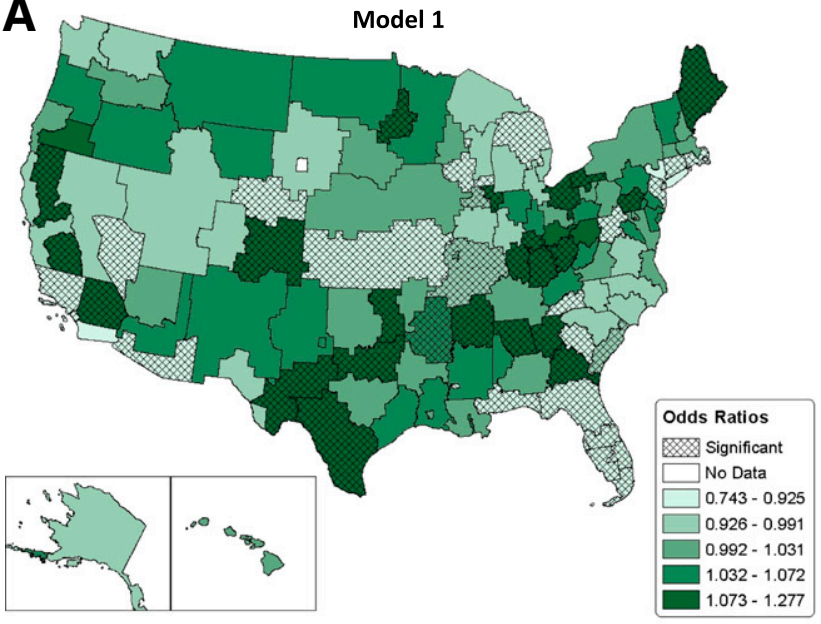

C
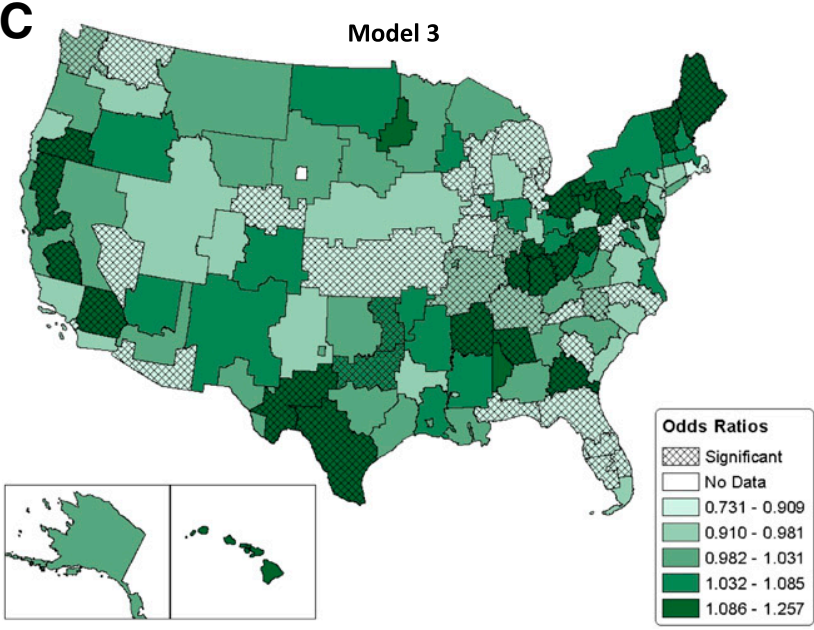

B

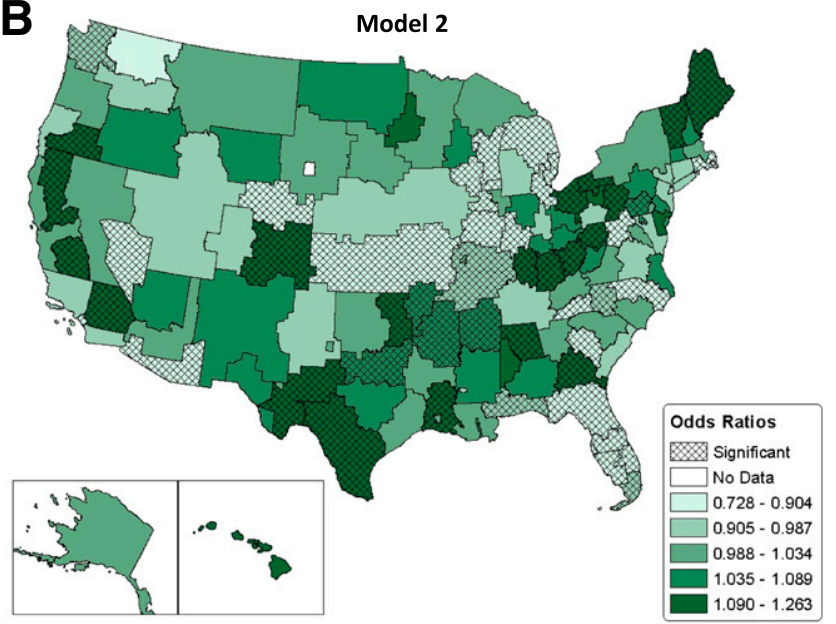

D

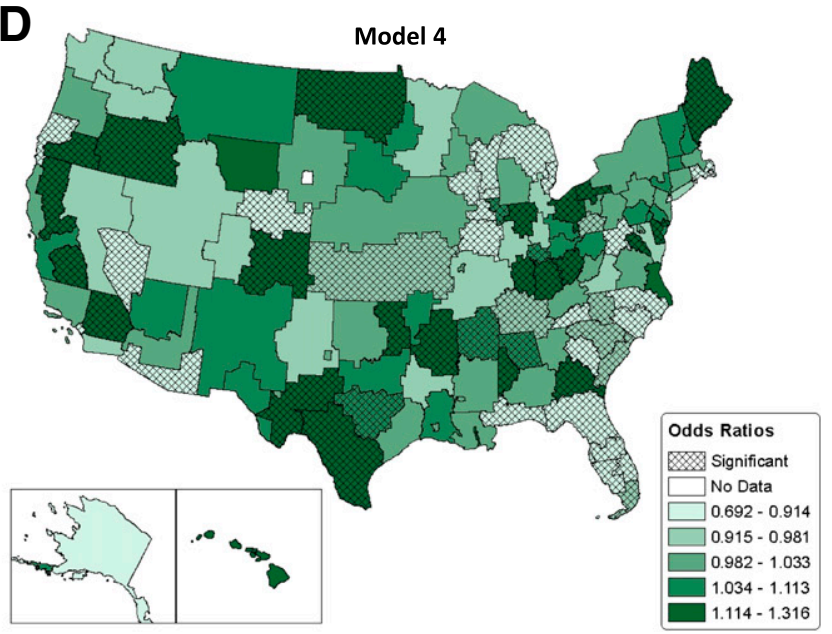

Figure 1-A-D: Spatial random-effects models for catchment area. Shading indicates quintiles of the adjusted ORs of uncontrolled diabetes in each catchment area relative to an average catchment area. Thus, ORs $>1$ have higher odds of uncontrolled diabetes relative to an average catchment area, and ORs $<1$ have lower odds of uncontrolled diabetes relative to an average area. Models are adjusted for covariates as indicated in Table 2.

with higher probability of uncontrolled diabetes in NHW had proportionally higher probability of uncontrolled diabetes in NHBs and Hispanics.

\section{Model 2}

Relative to NHWs, the odds of having uncontrolled relative to controlled diabetes were 1.10 -fold $(95 \% \mathrm{Cl} 1.08,1.12)$ higher in NHB and 1.35-fold (1.31, 1.39) higher in Hispanics after adjusting for demographic factors, comorbidity burden, catchment areas, and medication use (Table 2, model 2). Diabetes medication type had a strong association with uncontrolled diabetes, with odds of having uncontrolled relative to controlled diabetes 3.11-fold $(3.30,3.20)$ higher in those taking oral only, 12.0-fold (11.6, 12.3) higher in those taking insulin only, and 14.5-fold (14.1, 14.9) higher in those taking both oral and insulin relative to those not using diabetes medications. Medication treatment patterns were examined by catchment area (Supplementary Fig. 3A-D). Marked differences in medication treatment patterns were noted, with the percentage of patients not using any medication ranging from $23.2 \%$ to $49.6 \%$ depending on catchment area, with higher percentages of nonuse in the Northeast and Florida. Use of oral medications only (range 25.2-45.3\%), insulin only (6.9-17.5\%), and both oral medication and insulin (10.5-16.9\%) also varied by catchment area.

\section{Model 3}

Racial/ethnic group difference in diabetes control persisted after additionally adjusting for health care utilization and access metrics. Relative to NHWs, the odds of having uncontrolled relative to controlled diabetes were 1.11-fold (95\% $\mathrm{Cl} 1.09,1.14)$ higher in NHBs and 1.36- fold $(1.32,1.41)$ higher in Hispanics (Table 2, model 3).

\section{Model 4}

These racial/ethnic differences persisted after limiting the population to patients using diabetes medications and further adjusting for medication adherence (Table 2, model 4).

\section{Secondary Analyses}

When an aggressive $\mathrm{HbA}_{1 \mathrm{c}}$ cut point of $7 \%$ (53 mmol/mol) is used, NHB (OR 0.95 [95\% $\mathrm{Cl} 0.94,0.97]$ actually had a lower odds of suboptimal diabetes control than NHWs, while Hispanics (1.20 [1.17, 1.22]) remained at elevated but attenuated odds relative to NHWs. In comparison, when a conservative $\mathrm{HbA}_{1 \mathrm{c}}$ cut point of $9 \%$ was used, NHBs $(1.27[1.25,1.29])$ and Hispanics $(1.43[1.39,1.46])$ had substantially higher odds of suboptimal diabetes control than NHWs (Supplementary Table 1). 

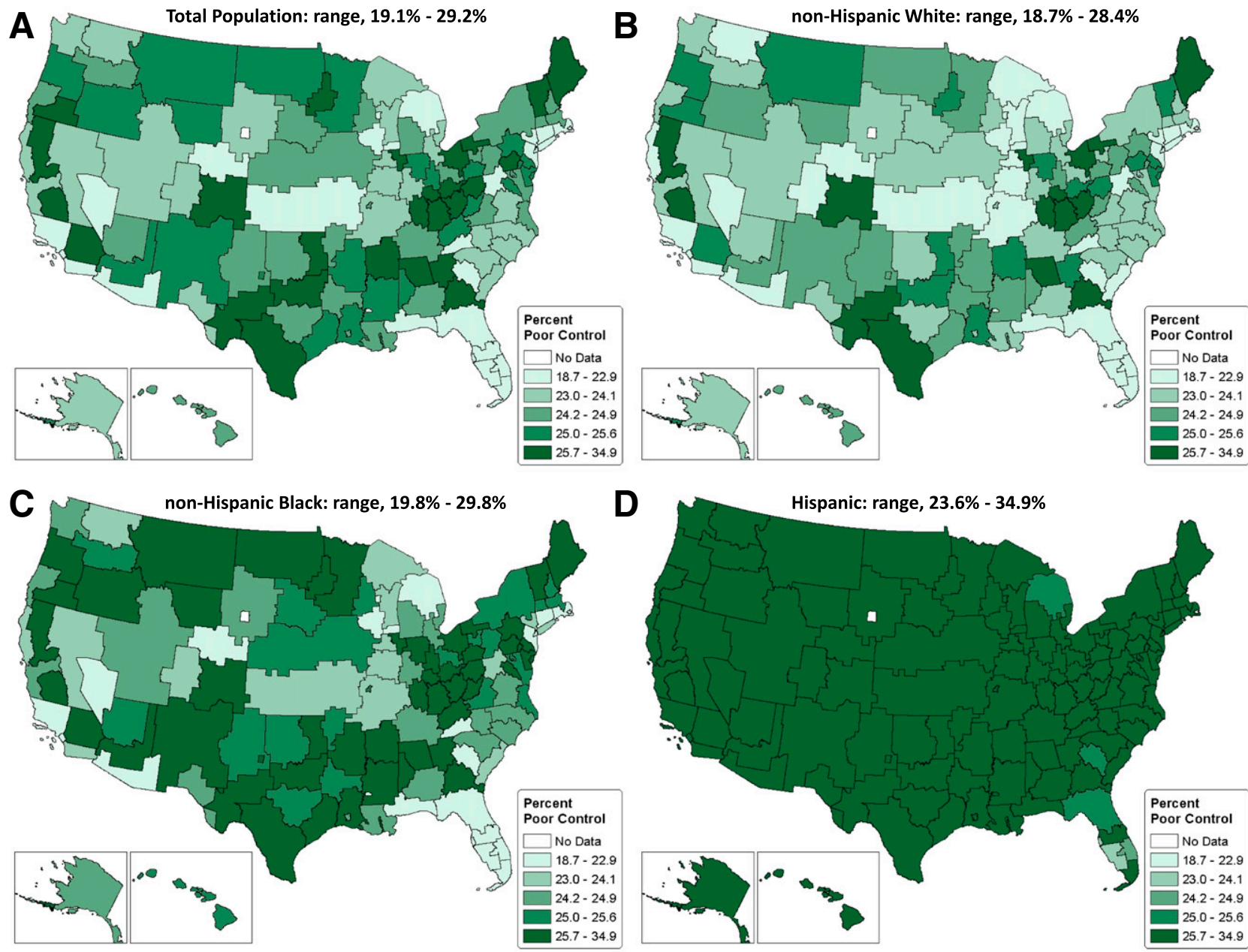

Figure 2-A-D: Predicted probability of suboptimal diabetes control by catchment area. Predictions are based on model 1 in Table 2 and correspond to individuals with average covariate values for age, sex, race, service-connected disability, marital status, and van Walraven comorbidity score.

\section{CONCLUSIONS}

In this study, we leveraged the largest integrated health care system in the U.S., the VHA, to examine the influence of space and race on uncontrolled diabetes. We asked whether there are differences in diabetes control at the level of the VAMC, whether racial/ethnic group influences uncontrolled diabetes, and whether there are disparities in experience and treatment causing racial/ ethnic group variation. Analyses within a Bayesian modeling framework identified significant geographic variation in suboptimal diabetes control after accounting for individual-level demographic factors, comorbidity burden, utilization, and access metrics as well as for diabetes medication use and adherence. This is a key finding that indicates that there are unmeasured geographic determinants not addressed in this study. Potential geographic determinants not examined include socioeconomic status and health care provider workforce differences between VAMCs and at the community level. Overall adjusted prevalence of uncontrolled diabetes ranged from $16 \%$ to $24 \%$ across VAMC catchment areas. This suggests that after controlling for multiple factors, the worst-performing catchment area had an additional $8 \%$ of patients with uncontrolled diabetes compared with the best area.

Similar to earlier studies (3-5), we found evidence of racial/ethnic group disparities in suboptimal diabetes control, with NHBs having a somewhat higher odds and Hispanics having a particularly higher odds of suboptimal control relative to NHWs. Moreover, variation in odds of diabetes control across VAMC catchment areas and across racial/ethnic groups was not diminished after adjusting for use and type of medication, both factors that varied substantially across VAMC catchment areas. Adjustment for utilization and access metrics, including outpatient visit frequency, distance to primary care, rural residence, and VAMC primary care wait time as well as for diabetes medication adherence, also failed to diminish variation in diabetes control across catchment areas or racial/ ethnic groups. These results are consistent with general findings reported in a systematic review of racial and ethnic disparities in the VA health care system (35).

Importantly, although VAMC catchment area and racial/ethnic group were significantly associated with uncontrolled diabetes, we did not find global evidence of a space-by-race interaction. In other words, relative racial/ethnic disparities in poor glycemic control appeared consistent across most, but not all, VAMC catchment areas. 
Table 2-Posterior OR (95\% CIs) for suboptimal relative to good glycemic control for covariates of interest

\begin{tabular}{|c|c|c|c|c|}
\hline & $\begin{array}{c}\text { Model } 1 \\
(n=1,140,634)\end{array}$ & $\begin{array}{c}\text { Model } 2 \\
(n=1,140,634)\end{array}$ & $\begin{array}{c}\text { Model 3 } \\
(n=1,140,634)\end{array}$ & $\begin{array}{c}\text { Model } 4^{*} \\
(n=815,255)\end{array}$ \\
\hline \multicolumn{5}{|l|}{ Race } \\
\hline NHW & 1.00 & 1.00 & 1.00 & 1.00 \\
\hline NHB & $1.07(1.05,1.09)$ & $1.10(1.08,1.12)$ & $1.11(1.09,1.14)$ & $1.11(1.09,1.14)$ \\
\hline Hispanic & $1.34(1.31,1.38)$ & $1.35(1.31,1.39)$ & $1.36(1.32,1.41)$ & $1.36(1.31,1.41)$ \\
\hline Age (1 year) ${ }^{\dagger}$ & $0.97(0.97,0.97)$ & $0.97(0.97,0.97)$ & $0.97(0.97,0.97)$ & $0.97(0.97,0.97)$ \\
\hline Male (female) & $1.37(1.31,1.43)$ & $1.23(1.16,1.29)$ & $1.20(1.14,1.26)$ & $1.24(1.18,1.30)$ \\
\hline Service-connected disability $\geq 50 \%$ & $0.95(0.93,0.96)$ & $0.80(0.79,0.82)$ & $0.84(0.82,0.85)$ & $0.84(0.83,0.86)$ \\
\hline Married (not married) & $0.90(0.89,0.91)$ & $0.96(0.94,0.97)$ & $0.94(0.93,0.95)$ & $0.93(0.91,0.94)$ \\
\hline van Walraven comorbidity score ${ }^{\dagger}$ & $1.00(1.00,1.00)$ & $0.98(0.98,0.98)$ & $0.98(0.98,0.98)$ & $0.98(0.98,0.98)$ \\
\hline \multicolumn{5}{|l|}{ Diabetes medications } \\
\hline No medications & - & 1.00 & 1.00 & - \\
\hline Oral only & - & $3.11(3.03,3.20)$ & $3.17(3.08,3.27)$ & 1.00 \\
\hline Insulin only & - & $11.95(11.63,12.32)$ & $12.51(12.07,12.90)$ & $4.01(3.92,4.12)$ \\
\hline Both oral and insulin & - & $14.46(14.09,14.86)$ & $15.44(14.99,15.92)$ & $4.65(4.56,4.74)$ \\
\hline Medication adherent (vs. nonadherent) & - & - & - & $0.85(0.83,0.86)$ \\
\hline \multicolumn{5}{|l|}{ Outpatient visit day frequency/year } \\
\hline $1-3$ & - & - & 1.00 & 1.00 \\
\hline $4-11$ & - & - & $0.89(0.86,0.92)$ & $0.95(0.91,0.99)$ \\
\hline $12-23$ & - & - & $0.82(0.80,0.85)$ & $0.94(0.89,0.97)$ \\
\hline$\geq 24$ & - & - & $0.71(0.69,0.74)$ & $0.93(0.89,0.96)$ \\
\hline \multicolumn{5}{|l|}{ Primary care driving distance (miles) } \\
\hline $\mathrm{Q} 1(\leq 6)$ & - & - & 1.00 & 1.00 \\
\hline Q2 (7-12) & - & - & $0.99(0.97,1.01)$ & $0.98(0.95,1.00)$ \\
\hline Q3 (13-23) & - & - & $1.00(0.98,1.03)$ & $1.00(0.97,1.021$ \\
\hline Q4 $(>23)$ & - & - & $0.98(0.96,1.01)$ & $1.01(0.88,1.03)$ \\
\hline Wait time primary care ( 1 day) & - & - & $1.01(1.01,1.01)$ & $1.01(1.00,1.01)$ \\
\hline Rural (vs. urban) & - & - & $1.03(1.01,1.05)$ & $1.03(1.01,1.05)$ \\
\hline Spatial variance & 0.05 & 0.05 & 0.04 & 0.06 \\
\hline
\end{tabular}

*Limited to those taking medication. TTight Cls are the result of very large sample size.

Our primary analyses used $\mathrm{HbA}_{1 \mathrm{c}} \geq 8 \%$ $\left(\mathrm{HbA}_{1 \mathrm{c}} \geq 64 \mathrm{mmol} / \mathrm{mol}\right)$ to define uncontrolled diabetes. In secondary analyses, we examined the impact of using both an aggressive $\left(\mathrm{HbA}_{1 \mathrm{c}} \geq 7 \%\right.$ [ $\geq 53 \mathrm{mmol} /$ $\mathrm{mol}])$ and a conservative $\left(\mathrm{HbA}_{1 \mathrm{c}} \geq 9 \%\right.$ [ $\geq 75 \mathrm{mmol} / \mathrm{mol}])$ cut point to define uncontrolled diabetes. Given the older age and high comorbidity burden in the VA, it was not surprising that $49.8 \%$ of veterans had uncontrolled diabetes using the aggressive cut point, while $12.3 \%$ had uncontrolled diabetes using the conservative cut point. Interestingly, spatial patterns across VAMC catchment areas remained similar regardless of $\mathrm{HbA}_{1 \mathrm{c}}$ cut point used, while racial/ethnic differences varied depending on cut point used. Namely, racial/ethnic differences were attenuated substantially when an $\mathrm{HbA}_{1 \mathrm{c}}$ cut point of $7 \%$ (53 mmol/mol) was used, while racial/ethnic differences were accentuated when an $\mathrm{HbA}_{1 \mathrm{c}}$ cut point of $9 \%$ (75 mmol/mol) was used.

Previous studies, especially work done in the VA, focused on controlling for spatial location through inclusion of terms for region or rural/urban residence rather than on taking spatial variability into account in the modeling approach $(5,11)$. Such strategies limit understanding of the role spatial distribution plays on outcomes and potentially confound assessments of disparities (25). The Centers for Disease Control and Prevention provides interactive maps, reports of regional variations in diabetes prevalence, and diabetes-related indicators (36). The Dartmouth Atlas documents variations in diabetes testing rates at the county, hospital referral region, and hospital service areas (37). However, we are unaware of analyses or reports of $\mathrm{HbA}_{1 \mathrm{c}}$ control rates. In this study, direct inclusion of a spatial effect in our models allowed us to map estimates for each VAMC catchment area and examine resulting geographic distributions across multiple modeling strategies. Overall, we found that the estimated spatial effects were very consistent across our models, a finding that indicates that location influences diabetes control after accounting for many individual-level factors. This is important because it suggests a geographic component to diabetes control that needs to be further explored.

Interestingly, patterns of uncontrolled diabetes within the VA did not mirror patterns of diabetes prevalence across the U.S. (38). Previous research has identified a "diabetes belt" that comprises 644 counties in the southern U.S. with diabetes prevalence rates in the adult population of $>11 \%$ (38). While high diabetes prevalence in the general population overlapped with suboptimal diabetes control in parts of Appalachia, Georgia, Alabama, Mississippi, and Tennessee, parts of the diabetes belt had lower-than-average rates of uncontrolled diabetes in the VA, indicating that areas of high diabetes prevalence can have below-average rates of uncontrolled diabetes. Identifying factors that contribute to diabetes control could lead to development of successful interventions to improve care across lower-performing 
VAMC catchment areas. Trivedi and Grebla (39) found that VA care was associated with $18.2 \%$ higher rates of LDL cholesterol control, $18.5 \%$ higher rates of $\mathrm{HbA}_{1 \mathrm{c}}$ control, and $21.6 \%$ higher rates of blood pressure control compared with Medicare Advantage, but they did not look at whether these effects varied geographically.

Strengths of our study include the large study population; our ability to map and analyze diabetes control at the VAMC catchment level and control for multiple factors while examining spatial variation in diabetes control rates; the extensive data available on comorbidities, diabetes medication use, and adherence that accounted for simultaneous use of multiple medications and utilization and access metrics (outpatient visit number, primary care driving distance, rural vs. urban residence, and primary care wait time); and the ability to identify racial/ethnic group in $95.5 \%$ of the cohort. Limitations include lack of information on plasma glucose levels, hypoglycemia, and diabetes duration; an inability to discriminate between type 1 and type 2 diabetes; using MPR as a proxy for medication adherence, especially in the case of insulin (although we and others have used similar methodology $[5,40])$; the limited number of women in the veteran population, which limits generalizability of results to women; use of the VA population, which may limit generalizability since racial/ethnic differences and barriers seen within the VA may be different than those seen outside the VA; and limitations to our definition of VAMC catchment area. Because there were counties with split utilization, $12 \%$ of patients routinely received care outside their assigned VAMC catchment area.

As the burden of diabetes continues to increase in the U.S. and throughout the world, it is imperative that we understand determinants of diabetes control. In our cohort of $>1.1$ million veterans with diabetes, more than one-quarter had uncontrolled diabetes despite decades of effective diabetes treatments, high access to medical care within the VA system, and routine monitoring of diabetes care quality metrics at VAMCs, including Healthcare Effectiveness Data and Information Set and VA Strategic Analytics for Improvement and Learning metrics. After controlling for demographic factors and comorbidity burden, the prevalence of uncontrolled diabetes in the VAMC catchment area with the poorest control rates was estimated to be as high as $28.4 \%$ for NHWs, $29.8 \%$ for NHBs, and $34.9 \%$ for Hispanics. Importantly, relative racial/ethnic disparities in suboptimal glycemic control appeared consistent across most, but not all, VAMC catchment areas. In summary, we used advanced spatial modeling techniques to pinpoint locations with elevated rates of uncontrolled $\mathrm{HbA}_{1 \mathrm{c}}$ and to identify factors contributing to diabetes control that could inform policy decisions.

Funding. This material is based upon work supported by the Department of Veterans Affairs, Office of Research and Development, Health Service Research and Development grant $5101 \mathrm{HX002299}$, and by U.S. National Library of Medicine grant R21-LM-012866.

The funding sources of this study did not play a role in the study design; collection, analysis, or interpretation of data; writing of the report; or decision to submit the manuscript for publication. The article represents the views of the authors and not those of the VA or Health Services Research and Development.

Duality of Interest. No potential conflicts of interest relevant to this article were reported. Author Contributions. K.J.H. and B.N. conceived and designed the study, researched data, analyzed and interpreted data, wrote the manuscript, reviewed and edited the manuscript, and supervised the study. M.D. analyzed and interpreted data, wrote the manuscript, and reviewed and edited the manuscript. J.P., J.B., M.F.G., E.M., and R.N.A. interpreted data and wrote the manuscript. K.J.H. and B.N. are the guarantors of this work and, as such, had full access to all the data in the study and take responsibility for the integrity of the data and the accuracy of the data analysis.

Prior Presentation. Parts of this study were presented at the Health Services Research and Development/Quality Enhancement Research Initiative (HSR\&D/QUERI) National Conference 2019, Washington, DC, 19-31 October 2019.

\section{References}

1. American Diabetes Association. Economic costs of diabetes in the U.S. in 2017. Diabetes Care 2018;41:917-928

2. Centers for Disease Control and Prevention. National Diabetes Statistics Report, 2020. Available from https://www.cdc.gov/diabetes/pdfs/data/ statistics/national-diabetes-statistics-report.pdf. Accessed 22 June 2020

3. Casagrande SS, Aviles-Santa L, Corsino L, et al. Hemoglobin A1c, blood pressure, and LDL-cholesterol control among Hispanic/Latino adults with diabetes: results from the Hispanic Community Health Study/Study of Latinos (HCHS/SOL). Endocr Pract 2017;23:1232-1253

4. Stark Casagrande S, Fradkin JE, Saydah SH, Rust KF, Cowie CC. The prevalence of meeting A1C, blood pressure, and LDL goals among people with diabetes, 1988-2010. Diabetes Care 2013;36: 2271-2279

5. Egede LE, Gebregziabher M, Hunt KJ, et al. Regional, geographic, and racial/ethnic variation in glycemic control in a national sample of veterans with diabetes. Diabetes Care 2011;34: 938-943

6. Brown T, Andrews GJ, Cummins S, Greenhough B, Lewis DA, Power A. Health Geographies: A Critical Introduction. Chichester, U.K., Wiley-Blackwell, 2017

7. Brown T, McLafferty S, Moon G. A Companion to Health and Medical Geography. Chichester, U.K., Wiley-Blackwell, 2010

8. Goonesekera SD, Yang MH, Hall SA, Fang SC, Piccolo RS, McKinlay JB. Racial ethnic differences in type 2 diabetes treatment patterns and glycaemic control in the Boston Area Community Health Survey. BMJ Open 2015;5:e007375

9. Piccolo RS, Duncan DT, Pearce N, McKinlay JB. The role of neighborhood characteristics in racial/ ethnic disparities in type 2 diabetes: results from the Boston Area Community Health (BACH) Survey. Soc Sci Med 2015;130:79-90

10. Baker J, White N, Mengersen K. Spatial modelling of type II diabetes outcomes: a systematic review of approaches used. R Soc Open Sci 2015;2:140460

11. Walker RJ, Neelon B, Davis M, Egede LE. Racial differences in spatial patterns for poor glycemic control in the Southeastern United States. Ann Epidemiol 2018;28:153-159

12. Andrus MR, Kelley KW, Murphey LM, Herndon KC. A comparison of diabetes care in rural and urban medical clinics in Alabama. J Community Health 2004;29:29-44

13. Weingarten JP Jr., Brittman S, Hu W, Przybyszewski C, Hammond JM, FitzGerald D. The state of diabetes care provided to Medicare beneficiaries living in rural America. J Rural Health 2006;22:351-358

14. Kirkbride $\mathrm{K}$, Wallace N. Rural health clinics and diabetes-related primary care for Medicaid beneficiaries in Oregon. J Rural Health 2009;25: 247-252

15. Voeks JH, McClure LA, Go RC, et al. Regional differences in diabetes as a possible contributor to the geographic disparity in stroke mortality: the Reasons for Geographic and Racial Differences in Stroke Study. Stroke 2008;39:16751680

16. Myers CA, Slack T, Broyles ST, Heymsfield $\mathrm{SB}$, Church TS, Martin CK. Diabetes prevalence is associated with different community factors in the diabetes belt versus the rest of the United States. Obesity (Silver Spring) 2017;25: 452-459

17. Herman WH, Dungan KM, Wolffenbuttel $\mathrm{BH}$, et al. Racial and ethnic differences in mean plasma glucose, hemoglobin A1c, and 1,5anhydroglucitol in over 2000 patients with type 2 diabetes. J Clin Endocrinol Metab 2009; 94:1689-1694

18. Kirk JK, D'Agostino RB Jr., Bell RA, et al. Disparities in HbA1c levels between AfricanAmerican and non-Hispanic white adults with diabetes: a meta-analysis. Diabetes Care 2006; 29:2130-2136

19. Herman WH, Ma Y, Uwaifo G, et al.; Diabetes Prevention Program Research Group. Differences in $\mathrm{A} 1 \mathrm{C}$ by race and ethnicity among patients with 
impaired glucose tolerance in the Diabetes Prevention Program. Diabetes Care 2007;30: 2453-2457

20. Ziemer DC, Kolm P, Weintraub WS, et al. Glucose-independent, black-white differences in hemoglobin A1c levels: a cross-sectional analysis of 2 studies. Ann Intern Med 2010;152:770777

21. Heisler M, Smith DM, Hayward RA, Krein SL, Kerr EA. Racial disparities in diabetes care processes, outcomes, and treatment intensity. Med Care 2003;41:1221-1232

22. Kerr EA, Gerzoff RB, Krein SL, et al. Diabetes care quality in the Veterans Affairs Health Care System and commercial managed care: the TRIAD study. Ann Intern Med 2004; 141:272-281

23. Department of Veterans Affairs; Department of Defense. VA/DoD Clinical Practice Guidelines for the Management of Diabetes Mellitus in Primary Care. Washington, DC, Veterans Health Affairs. Department of Defense, 2017

24. American Diabetes Association. 6. Glycemic targets: Standards of Medical Care in Diabetes2019. Diabetes Care 2019;42(Suppl. 1):S61S70

25. Department of Veterans Affairs Office of Health Equity. National Veteran Health Equity Report-FY2013. Washington, DC, Veterans Health Administration Office of Health Equity. Available from https://www.va.gov/HEALTHEQUITY/
docs/National_Veterans_Health_Equity_Report FY2013_FINAL_508_Comp.pdf. Accessed 22 June 2020

26. Quan $H$, Sundararajan V, Halfon $P$, et al. Coding algorithms for defining comorbidities in ICD-9-CM and ICD-10 administrative data. Med Care 2005;43:1130-1139

27. van Walraven $C$, Austin $P C$, Jennings $A$, Quan $\mathrm{H}$, Forster AJ. A modification of the Elixhauser comorbidity measures into a point system for hospital death using administrative data. Med Care 2009;47:626-633

28. Lau DT, Nau DP. Oral antihyperglycemic medication nonadherence and subsequent hospitalization among individuals with type $2 \mathrm{di}$ abetes. Diabetes Care 2004;27:2149-2153

29. Kirkman MS, Rowan-Martin MT, Levin R, et al. Determinants of adherence to diabetes medications: findings from a large pharmacy claims database. Diabetes Care 2015;38:604-609 30. Phibbs CS, Cowgill EH, Fan AY. Guide to the PSSG Enrollee File. Guidebook. Menlo Park, CA, VA Palo Alto, Health Economics Resource Center, 2015

31. Veterans Health Administration. 2015 Local Patient Access Data. Available from www.va.gov/ health/access-audit.asp. Accessed 22 June 2020 32. Banerjee S, Carlin BP, Gelfand AE. Hierarchical Modeling and Analysis for Spatial Data. 2nd ed. Boca Raton, FL, CRC Press/Chapman \& Hall, 2015
33. R Foundation for Statistical Computing. The R Project for Statistical Computing. Available from https://www.R-project.org/. Accessed 22 June 2020

34. Gelman J, Carlin JB, Stern HS, Dunson DB, Vehtari A, Rubin DB. Bayesian Data Analysis. 3rd ed. Chapman and Hall/CRC, 2013

35. Saha SFM, Toure J, Tippins K, Weeks C. Racial and Ethnic Disparities in the VA Healthcare System: A Systematic Review. Department of Veteran Affairs Health Services Research and Development Service, 2007

36. Kirtland KA, Burrows NR, Geiss LS. Diabetes Interactive Atlas. Prev Chronic Dis 2014;11: 130300

37. Wennberg JE, Fisher ES, Goodman DC, Skinner JS, Bronner KK. Tracking the Care of Patients with Severe Chronic Illness-the Dartmouth Atlas of Health Care 2008. The Trustees of Dartmouth College, 2008

38. Barker LE, Kirtland KA, Gregg EW, Geiss LS, Thompson TJ. Geographic distribution of diagnosed diabetes in the U.S.: a diabetes belt. Am J Prev Med 2011;40:434-439

39. Trivedi AN, Grebla RC. Quality and equity of care in the veterans affairs health-care system and in Medicare advantage health plans. Med Care 2011;49:560-568

40. Cramer JA, Pugh MJ. The influence of insulin use on glycemic control: how well do adults follow prescriptions for insulin? Diabetes Care 2005;28:78-83 